\title{
La couleur comme expérience synesthésique dans l'Antiquité
}

Colour as Synaesthetic Experience in Antiquity

\section{Mark Bradley}

Traducteur : Lydia Pelletier-Michaud

\section{OpenEdition \\ Journals}

\section{Édition électronique}

URL : http://journals.openedition.org/mythos/627

DOI : $10.4000 /$ mythos. 627

ISSN : 2037-7746

\section{Éditeur}

Salvatore Sciascia Editore

\section{Édition imprimée}

Date de publication : 1 décembre 2017

Pagination : 95-112

ISSN : 1972-2516

\section{Référence électronique}

Mark Bradley, «La couleur comme expérience synesthésique dans l'Antiquité », Mythos [En ligne], 11 | 2017, mis en ligne le 24 septembre 2019, consulté le 28 septembre 2019. URL : http:// journals.openedition.org/mythos/627 ; DOI : 10.4000/mythos.627 


\section{La couleur comme expérience synesthésique dans l'Antiquité}

\section{Mark Bradley}

\section{Résumé}

La couleur est bien plus que des ondes lumineuses atteignant la rétine. Dans les cercles philosophiques de l'Antiquité, elle était décrite comme le principal objectif de la vision : c'était la " peau " extérieure d'un objet, rendant celui-ci visible ou " sensible " au spectateur. Et cependant, la littérature gréco-romaine est remplie d'exemples de catégories de couleur qui ne font pas sens uniquement en termes visuels : la " mer sombre comme le vin " (oinops pontos), la peau couleur " petit-lait " (orōdēs) dans la médecine hippocratique, les visages rougissants (rubens), les cheveux couleur de miel (mellei crines), la peau de marbre des jeunes filles dans l'élégie latine, les vêtements couleur safran des orientaux décadents, les robes luxueuses de pourpre à l'odeur de poisson de la cour impériale... La couleur sollicite non seulement la vue, mais aussi l'odeur, le toucher et le goût. Le présent article développera des arguments et des idées présentées dans la monographie de l'auteur Colour and Meaning in Ancient Rome (Cambridge University Press, 2009) : dans les sociétés prémodernes comme la Grèce et Rome, les couleurs, en raison de leurs liens étroits avec des objets spécifiques et des phénomènes (plutôt que simplement des parties du spectre), étaient fréquemment des expériences synesthésiques qui avaient trait à de multiples expériences sensibles. En partant de cas particuliers, l'auteur montrera que la couleur était une expérience multi-sensorielle et que la nature de cette expérience était une préoccupation de la pensée ancienne dans plusieurs aires d'activités dans le monde gréco-romain. La compréhension de ce phénomène ne nous aide pas seulement à traduire les termes de couleurs grecs et latins. La couleur était une unité d'information sensorielle de base à travers laquelle les Anciens expérimentaient et évaluaient le monde autour d'eux. La collaboration des sens dans ces expériences invite à une approche de la perception, de la connaissance et de la compréhension qui pourrait être différente de celle employée pour le monde occidental moderne.

\section{Abstract}

Colour is about more than just lightwaves hitting the retina. In ancient philosophical circles, colour was described as the primary object of vision: it was the external 'skin' that existed at the surface of an object, and what made the object visible or 'sensible' to a viewer. And yet, Greco-Roman literature is riddled with examples of colour categories that do not make sense simply in visual terms: from Homer's 'winedark sea' (oinops pontos) to 'whey-coloured' (orōdēs) skin in Hippocratic medicine, from blushing (rubens) faces to the honey-coloured hair (mellei crines) and marbled skin of coveted girls in Augustan elegy, and from the saffron garments of decadent easterners to the expensive fishy-smelling purple robes of the lateantique imperial court, colours appealed not just to sight, but also to smell, touch and taste. This paper will use arguments and ideas explored in my monograph Colour and Meaning in Ancient Rome (Cambridge University Press, 2009) to suggest that colours in pre-modern societies such as Greece and Rome, because of their close ties to specific objects and phenomena (rather than just parts of the spectrum), were frequently synaesthetic experiences which appealed to multiple senses and mobilized more than just eyesight. I will draw upon case studies from a range of genres and contexts to demonstrate not only that colour was a multi-sensory experience, but also that the nature of this experience was a central preoccupation of ancient thought in several areas of activity in the Greco-Roman world. An understanding of this principle, then, can do more than just help us to translate and understand difficult Greek and Latin colour terms: colour was a basic sensory unit of information through which ancients experienced and evaluated the world around them, and the collaboration of the senses in these experiences suggests an approach to perception, knowledge and understanding which could be very different from that employed in the modern west.

\section{Mots clefs}

Couleur • synesthésie - polysensoriel • traduction • perception

\section{Keywords}

Colour - synaesthesia - multisensory - translation • perception tique homérique reposait exclusivement sur l'opposition entre clair et foncé, supposant 
que l'organe de la vision "n'était que sommairement développé chez les Grecs »" Il n'était pas le premier à soutenir de telles affirmations à propos d'une vision des couleurs déficiente chez les Grecs : une génération plus tôt, l'érudit allemand Goethe avait émis de semblables remarques à propos de l'art antique, et d'autres avaient souligné la pauvreté matérielle des couleurs dans l'environnement méditerranéen antique - la variété limitée des teintures, des peintures et des fleurs, de même que la relative uniformité de la couleur des corps anciens. Au cours du XIX siècle, ces idées entrèrent en résonance avec certaines théories de Darwin à propos de l'évolution humaine et du développement de la civilisation, et certains allèrent jusqu'à évoquer le phénomène du daltonisme, récemment découvert, pour expliquer le manque de sensibilité des Anciens aux couleurs ${ }^{2}$. Depuis, la question de la pauvreté et de l'imprécision du vocabulaire de la couleur dans les langues anciennes fait toujours l'objet de débats animés parmi les philologues et les anthropologues; au cours des derniers siècles, les études portant sur la vision des couleurs dans l'Antiquité ont été dominées par les efforts visant à démontrer que les Anciens employaient au contraire un système de couleurs très complexe. Suivant l'argument habituel, ce système aurait opéré selon des paramètres quelque peu différents des nôtres : plutôt que la nuance, les Anciens auraient avant tout été sensibles à des critères tels que la luminosité, la saturation et la texture, ou encore à des variables encore plus inattendues, telles que l'odeur, le mouvement et la fluidité ${ }^{3}$.

Les Anciens, bien entendu, n’étaient certes pas "daltoniens ", et il importe d'user de tout autant de prudence lorsque nous employons le terme "synesthésie " pour décrire leur expérience sensible. Comme chacun sait, la synesthésie désigne une disposition idiosyncratique du cerveau lors de laquelle la stimulation d'une voie sensorielle fait naître automatiquement et de façon involontaire des expériences impliquant un second canal sensoriel ${ }^{4}$. Selon cette définition, les Anciens n'étaient pas davantage synesthètes que daltoniens. Cela ne doit pas nous empêcher de nous demander comment et pourquoi des associations entre les différents sens avaient lieu dans la pensée ancienne, ni de remettre en question la distinction entre les cinq sens que la plupart d'entre nous considère aujourd'hui comme allant de soi ${ }^{5}$.

Cet article se propose d'explorer les approches de la couleur au-delà du paradigme visuel en examinant une variété d'expériences anciennes de la couleur qui peuvent apparaître comme "synesthésiques ». L'une des approches possibles consisterait à étudier un ensemble de contextes anciens au sein desquels la couleur paraît être utilisée métaphoriquement pour renvoyer à d'autres expériences sensibles, comme par exemple la théorie musicale grecque classique, avec ses gammes « chromatiques ", ou encore les colores de l'art oratoire romain ${ }^{6}$. Ce type d'expériences pourrait fort bien nous permettre d'établir une histoire de la synesthésie : pour

1 Gladstone 1858, 488, dans le chapitre «Homer's Perceptions and Use of Colour », commenté par IRWIN $1974,6-7$.

2 Cf. en particulier Schultz 1904, 187-188.

3 La question de la nature du système des couleurs dans l'Antiquité est toujours amplement débattue. Pour plus de détails, voir Bradley 2009, 12-17; Grand-Clément 2011, 11-19. Pour une étude détaillée de deux catégories de couleur grecques particulièrement problématiques (avec des réserves sur ses conclusions quelque peu simplistes), voir Maxwell-Stuart 1981.

4 À propos des diverses approches artistiques et scientifiques de la synesthésie, voir CAMPEN 2008.

5 Howes 2006.

6 À propos de la " couleur musicale " grecque, voir LANDEls 1999, 90-92 et BARKER 1984, 225-227 (entre autres); sur le color rhétorique latin, voir BradLey 2009, chapitre 4 ; cf. également p. 69-70, sur les concepts grecs de "couleur» (chrōma) dans l'art oratoire. 
les experts de l'harmonie musicale, certains accords correspondent à des sensations de couleur, et ce de manière comparable à la corrélation qui s'établit entre musique et couleurs dans l'esprit de véritables synesthètes de l'Occident moderne ${ }^{7}$. Les orateurs romains ont d'ailleurs, dès la fin de la République, développé des discours dans lesquels le style rhétorique, les arguments et les ornements étaient intimement liés aux couleurs naturelles et artificielles du corps. Il existe toutefois une approche susceptible de nous permettre d'établir un lien encore plus direct entre les couleurs et les autres expériences sensibles dans l'Antiquité, et celle-ci implique de se pencher sur des questions essentielles : pour les Anciens, qu'était une couleur? Quel rôle jouait la couleur dans la relation entre l'observateur et le monde qui l'entourait ? Comment faisait-on l'expérience de la couleur, et selon quels critères l'appréciait-on?

\section{Couleurs et cultures}

Deux arguments suffisent à prouver que l'étude des couleurs dans l'Antiquité mérite l'attention du milieu académique. En premier lieu, de nombreux termes anciens sont notoirement difficiles à traduire : il existe une longue liste de termes grecs et latins que l'on ne peut rendre directement en anglais ; en outre, un nombre considérable de couleurs "simples » employées aujourd'hui semblent n'avoir eu aucun équivalent dans l'Antiquité. Les couleurs des Anciens ne semblent pas non plus toujours pertinentes selon nos propres repères sensibles. Nous devons nous efforcer de comprendre de manière plus approfondie la manière dont les poètes, les artistes, les orateurs, les philosophes et les historiens ont employé des catégories de couleurs et formulé la relation entre leur art ou leurs écrits et le monde qu'ils décrivaient. La seconde raison confirmant l'importance de la couleur est le fait que celle-ci est formulée et catégorisée d'une manière propre à chaque communauté qui en fait usage.

Dans un article stimulant publié en 1985 et intitulé « How Culture Conditions the Colours We See » («L'influence de la culture sur notre vision des couleurs »), Umberto Eco propose un diagramme illustrant à quel point les couleurs en anglais, en latin et en hanunóo fonctionnent différemment. Les Hanunóo, un groupe ethnique d'une province des Philippines, sont connus depuis longtemps pour utiliser un système complexe de termes de couleur qui non seulement recoupe et intègre des qualités telles que l'humidité, la texture et l'éclat, mais en outre fonctionne selon deux niveaux distincts, en fonction du type de communication mis en œuvre ${ }^{8}$. Bien que l'usage d'un diagramme de ce type - impliquant de quantifier l'expérience de la couleur en fonction de la longueur d'onde - soit limité (l'interprétation des termes de couleur latins y est manifestement erronée), celui-ci montre bien comment la couleur peut, d'une culture à l'autre, mobiliser un ensemble très différent de critères esthétiques.

\begin{tabular}{|c|c|c|c|c|c|c|}
\hline $\mathrm{m} \mu$ & Average English & Latin & \multicolumn{3}{|c|}{ Hanunóo level 1} & Hanunóo level 2 \\
\hline $800-650$ & Red & us & Marara & $\tilde{E}$ & & \\
\hline $640-590$ & Orange & & & & 产: & \\
\hline $580-550$ & Yellow & & & & 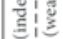 & \\
\hline $540-490$ & Green & & & 2 & 焉涪 & \\
\hline $480-460$ & Blue & & & 起 & $\sum_{\dot{\alpha}}^{n} ! \sum_{\dot{\alpha}}$ & \\
\hline $450-440$ & Indigo & & $\begin{array}{l}\text { Mabi:ru } \\
\text { (rotten) }\end{array}$ & 롱 & 总食 & \\
\hline $430-390$ & Violet & & & $\sum^{\pi}$ & $2 ! \frac{\pi}{2}$ & \\
\hline
\end{tabular}

Fig. 1. Diagramme comparatif des catégories de couleur en anglais, en latin et en hanunóo, incluant les longueurs d'ondes approximatives (d'après ECO 1985, commenté dans Bradley 2009, 25). Reproduit d'après l'original.

7 Voir à ce propos Rosen 2013, 96.

8 Voir Conklin 1955. 
La couleur, dès lors, constitue une composante élémentaire culturelle à l'aune de laquelle nous pouvons jauger la manière dont les humains voient le monde qui les entoure, et elle concerne bien davantage que de simples longueurs d'ondes atteignant la rétine. C'est un fait bien connu : différentes cultures peuvent distinguer et décrire les couleurs de manière distincte, et il existe de nombreux exemples de langues maniant la couleur selon des schémas inhabituels : par exemple, le russe possède deux termes distincts pour nommer la couleur que nous appelons " bleu ", tandis que le concept japonais ao recoupe le vert et le bleu. Diverses communautés africaines, sud-américaines et asiatiques emploient des systèmes de couleur qui sont pour nous fort étranges, incluant des propriétés et des qualités qui franchissent les domaines de l'odorat, du goût, du toucher et même du son ; les Hanunóo en sont un exemple bien connu. Dans le Soudan du Sud, une tribu nomade appelée Dinka a capté l'attention des anthropologues et des sociologues en raison de son usage inhabituel de la couleur : on a souvent affirmé que le riche répertoire de catégories de couleur de cette communauté comprenait des dizaines de termes dans la gamme des jaunes et des marrons, peut-être à cause de l'environnement aride dans lequel elle évolue (ce qui rappelle les Innus et leur vocabulaire varié pour les flocons de neige $)^{9}$. En fait, les recherches les plus récentes ont pu établir de manière incontestable que la perception d'un grand nombre de ces couleurs renvoyait non pas à des points précis sur nos nuanciers, mais à la robe (couleur, marques et taches) des vaches qui jouent un rôle si crucial dans la vie des Dinka. Cela signifie que le monde perçu par les Dinka - du moins le monde des chansons tribales, dans lesquelles on rencontre le plus souvent ces couleurs - est reformulé de manière à connecter intimement ses divers éléments avec ces créatures qui vivent, respirent, empestent et mugissent, auxquelles la communauté accorde une si grande valeur ${ }^{10}$. Il n'est pas anodin que dans leur ouvrage influent (bien qu'aujourd'hui largement discrédité) Basic Color Terms (1969), qui proposait un système de distinction des couleurs fondé sur l'évolution et le développement culturel, la complexité du langage de la couleur des Dinka ait complètement échappé à Brent Berlin et Paul Kay : ces auteurs, qui situaient de nombreuses communautés tribales "primitives" au stade I, le grec homérique au stade IIIb et (sans surprise) l'anglais, le japonais et le russe au stade VII, ont élevé le langage des Dinka aux sommets vertigineux du stade VII, car celui-ci leur semblait employer des couleurs pouvant correspondre à celles utilisées dans l'Occident développé. Comme Umberto Eco avec son diagramme, Brent Berlin et Paul Kay ont ainsi, de manière fortuite, mis en lumière les limites du présupposé faisant de la couleur un phénomène purement visuel pouvant systématiquement être répertorié sur nos nuanciers.

En fait, comme le montrent les Dinka (ainsi que les Innus avec leurs flocons de neige), les communautés distinguent des couleurs qui leur sont importantes ou signifiantes. Souvent, ces couleurs sont ancrées dans le monde matériel sensible et concret plutôt que fondées sur la seule apparence. Des études portant sur la vision des couleurs chez les primates ont montré que ceux-ci distinguaient le jaune, le vert et le rouge, qui correspondent respectivement aux fruits mûrs, immatures et toxiques : pour eux, la couleur est intimement liée au monde des goûts et

9 À propos de ce mythe, voir Lienhardt 1960, 12-16 et BEARD 2002, 47.

10 À propos du système chromatique dinka pour les bovins, voir Coote 1992, 250. Pour une réponse à l'approche de J. Coote, voir Gell 1995 ; selon ce dernier, le répertoire chromatique en question appartient au langage des poètes dinka et non à la communauté en général. Voir également BradLeY 2009, 29-30. Sur le système des couleurs associées au bétail chez les Mursi en Éthiopie, voir EczET 2016. 
des odeurs. La couleur, dès lors, n'est pas un phénomène objectif : ces mêmes études énoncent d'ailleurs souvent en préface le principe cartésien selon lequel la couleur se situe dans l'esprit, et non dans le monde physique ${ }^{11}$.

Le présent article ne prétend pas démontrer qu'il est possible de formuler, à propos de la couleur dans l'Antiquité, des conclusions aussi affirmatives que celles que nous pouvons tirer à propos des Dinka ou des singes frugivores ; il est toutefois manifeste que nous avons beaucoup à apprendre sur les valeurs et les priorités des Anciens en examinant la manière dont ils percevaient la couleur, la décrivaient et formulaient sur elle des discours. Nous allons débuter en nous interrogeant sur ce que les penseurs de l'Antiquité considéraient comme étant une couleur. La question de la définition de la couleur dans l'Occident moderne n'est certes pas simple, mais l'on s'accorde en général pour dire que nous procédons au moyen d'un système de couleurs essentiellement abstrait. Nous pouvons concevoir la couleur comme quelque chose de distinct des gens, des objets et des paysages qu'elle colore : nous pouvons nous représenter en pensée ce qu'est « le jaune » et simplement transférer cette qualité des cheveux blonds à la paille, au soufre, aux teintures synthétiques ou aux stylos-feutres; notre "vert " peut décrire des plantes, des perroquets, des émeraudes, des visages malades, et ainsi de suite. Dans l'Antiquité, les couleurs semblent avoir opéré de manière bien différente.

\section{Aborder la couleur dans l'Antiquité}

Larticle de Michael Clarke, "The Semantics of Colour in the Early Greek Word Hoard " ("Principes sémantiques de la couleur dans le vocabulaire grec ancien »), présente l'une des études plus fines et des plus achevées portant sur la terminologie grecque de la couleur ${ }^{12}$. Cet auteur soutient qu'il existait, à la base de l'expérience ancienne de la couleur, un archétype sémantique : il s'agissait de pivots (ou "points de référence cognitifs ") autour desquels s'organisaient en cercles concentriques les diverses expériences de la couleur telles que vécues par les Grecs. L'étude de Michael Clarke se concentre sur quatre termes de couleur complexes : chlöros (interprété comme "vert / fécond / humide "), argos ("blanc étincelant / vif "); porphureos ( violet / bouillonnant»); et oinops ("sombre comme le vin / délirant / dionysiaque »).

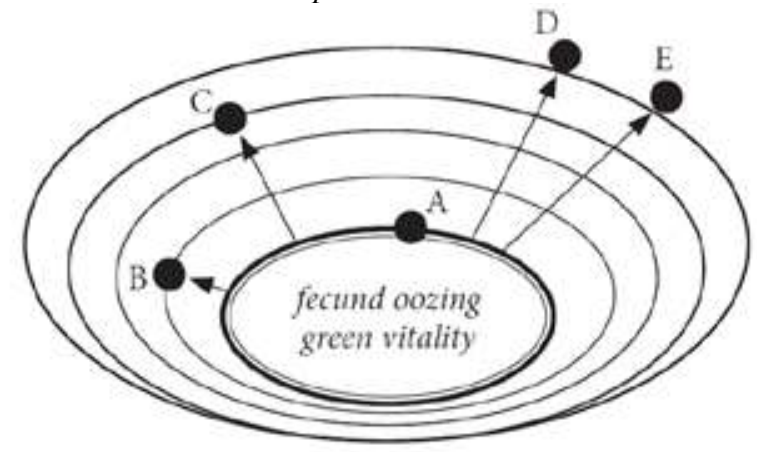
A. Plant
B. Dew
C. Cheese
D. River water
E. Tears

Fig. 2 : "Vitalité verte, humide et féconde ", tiré de Clarke 2004, illustration 2. Reproduit d'après l'original. A. Plante ; B. Rosée ; C. Fromage ; D. Eau d'une rivière ; E. Larmes.

11 Voir par exemple Regan et al. 2001.

12 Clarke 2004. 
La figure 2 montre comment, selon Michael Clarke, fonctionnait l'emploi du terme chlōros en grec archaïque : cette catégorie, qui évoque en essence les qualités d'une "vitalité verte, humide et féconde ", pouvait être appliquée à une variété d'objets ou de phénomènes de la nature présentant des qualités apparentées ; au cours du temps, ces associations seraient devenues plus flottantes, de sorte que ce qui était à l'origine une catégorie descriptive propre aux plantes pût par la suite s'élargir à des phénomènes s'y rattachant de manière plus vague, comme l'eau d'une rivière ou les larmes. L'approche de Michael Clarke fournit un modèle élaboré, à la pointe de la linguistique moderne : l'idée selon laquelle les anciens " archétypes » de couleurs se situaient souvent "à la jonction de plusieurs domaines cognitifs » que l'on a l'habitude de distinguer dans l'Occident moderne - la couleur, la lumière, le mouvement, l'état d'esprit, etc. - offre une solution convaincante pour mieux comprendre certaines des catégories de couleurs les plus déroutantes de l'Antiquité.

La recherche présentée dans cet article présente certains points communs avec l'approche de Michael Clarke : nous y défendons aussi l'idée selon laquelle les catégories archaïques étaient d'abord et avant tout liées à des expériences et ont ensuite été, avec le temps, appliquées de manière plus libre et créative à d'autres phénomènes. Cependant, plutôt que de considérer que l'expérience de la couleur par les Anciens était centrée sur un modèle abstrait, nous soutenons que les couleurs étaient à l'origine associées à des objets spécifiques et distincts : ainsi, chlōros ne fait pas référence à un vert abstrait, fécond et humide, mais signifie essentiellement "verdoyant » ou " couleur de plante »; oinops se rapporte directement au vin et dès lors, par extension, à sa couleur, sa saveur, ses effets et autres connotations; et ainsi de suite. Les implications de cette approche sont dès lors évidentes : en effet, une expérience de la couleur centrée sur l'objet peut aussi nous aider à comprendre pourquoi nous rencontrons si souvent, dans l'Antiquité, des usages de la couleur faisant appel aux autres sens.

Donc, lorsque les Romains pensent à uiridis (ou à chlōros), ils pensent à l'aspect des plantes et de la végétation plutôt que simplement à un vert abstrait : c'est pour cette raison que Columelle peut évoquer le " goût vert " (uiridis sapor) des olives, qu'Horace peut imaginer des "flammes vertes " (uirens flamma) surgissant de l'Etna, et qu'Aulu-Gelle peut qualifier un son fort et vif, tel le « $\mathrm{h}$ » aspiré, de uiridis ${ }^{13}$. À partir du moment où nous acceptons que le terme latin flauus signifie d'abord et avant tout «blond " plutôt que jaune, nous pouvons mieux comprendre pourquoi les poètes latins peuvent, de manière imagée, dire de champs de blé ondulants et de guirlandes de feuilles d'oliviers qu'ils sont flaui : ces réalités possèdent des longueurs d'onde semblables, mais ils partagent aussi des qualités tactiles et des connotations similaires ${ }^{14}$. Et si nous reconnaissons que caeruleus ne signifie pas simplement "bleu " mais évoque plutôt les profondeurs marines, nous comprenons mieux pourquoi les nuages d'orage sont souvent caerulei tandis qu'un ciel bleu clair ne l'est jamais, et pourquoi la caerula Crete n'est pas une île "bleue ", mais bien une île associée aux eaux profondes qui l'entourent ${ }^{15}$.

Cette idée de couleur en tant qu'expérience centrée sur l'objet cadre bien avec diverses approches philosophiques de la perception. Les penseurs grecs et romains issus de diverses écoles

13 Columelle, De l'agriculture 12, 49, 8 ; Horace, Épodes 17, 33 ; Aulu-Gelle, Nuits Attiques 2, 3, 2. Pour des remarques plus développées à propos de uiridis, voir Bradley 2009, 7-9.

14 À propos de flauus, voir Bradley 2009, 1-6. Pour les champs de blé flaui, cf. Tibulle 2, 1, 48 (deponit flauas annua terra comas); et pour les feuilles d'olivier flaui, cf. Virgile, Énéide 5, 309.

15 Bradley 2009, 9-11. Pour caerula Crete, cf. Sénèque, Hercule sur l'Eta, 1874. 
philosophiques ont fréquemment décrit la couleur (chrōma ou color) comme la surface extérieure ou la " peau " d'un objet, ce qui délimite cet objet et le rend visible. Dans le traité $D e$ l'âme $(2,132)$, Aristote fait remarquer que la couleur n'existe qu'à la surface d'un objet (ทँ $\pi \varepsilon \rho$

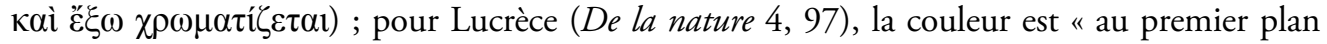
et tout à la surface " (in promptu quoniam est in prima fronte locata). Les termes grecs chröma, chroia et chrōs semblent approcher le sens de notre "couleur ", dans la mesure où ils font d'abord référence à la surface, en particulier à la peau d'un corps ou d'un visage (bien que leur sens ait parfois été élargi à la description des peintures, des teintures et des produits cosmétiques) ${ }^{16}$. Le terme latin color était porteur de connotations similaires. Les qualités auxquelles ces mots faisaient référence étaient dès lors inextricablement liées à l'extériorité et au superficiel - à la "surface "-, et c'est à partir de ce point que nous pouvons le mieux introduire notre hypothèse à propos des propriétés synesthésiques des couleurs dans l'Antiquité ${ }^{17}$. Dans la pensée ancienne, les couleurs fonctionnaient comme un répertoire rudimentaire du monde environnant. Dans les cercles philosophiques, la couleur était parfois décrite comme l'objet premier de la vision ${ }^{18}$; toutefois, parce qu'elle était si étroitement rattachée aux objets réels du monde, elle pouvait aussi faire appel à toute la gamme des sensations. Nous allons maintenant examiner divers contextes dans lesquels s'applique ce type d'expérience, centrée sur l'objet.

\section{La mer "sombre comme le vin" 19}

La mer "sombre comme le vin " d'Homère (oinops pontos) est sans doute le mieux connu des problèmes de la couleur dans l'Antiquité ${ }^{20}$. C'est la mer vers laquelle Achille, en deuil, tourne ses regards lors des funérailles de Patrocle (Iliade 23, 143), celle également dans laquelle Ulysse perd son vaisseau et ses hommes après le massacre des troupeaux du Soleil (Odyssée 5, $132 ; 5,221 ; 7,250 ; 12,388)$; l'épithète est employée à plusieurs autres occasions dans les vers d'Homère. Comme l'action, dans plusieurs de ces exemples, paraît se dérouler vers le moment du coucher du soleil, une hypothèse voulant que l'expression renvoie au rouge du soleil couchant a rencontré beaucoup de succès ces dernières années ; mais cette interprétation, obstinément visuelle, implique que la qualité " rouge " puisse être directement transférée du vin (qui peut être de plusieurs couleurs) aux couchers de soleil rougeoyants ${ }^{21}$. D'autres interprétations incluent l'effervescence de la fermentation, une surface chatoyante similaire à celle qu'évoque le terme porphyreos, tout aussi complexe (voir infra), voire même un type de vin bleuâtre, de mauvaise qualité, comparable à ceux qu'évoquent les expressions désuètes " petit bleu / gros bleu "22. Mais toutes ces interprétations s'appuient, encore une fois, sur un éventail d'associations sensorielles abstraites. Plus récemment, comme nous l'avons mentionné plus

16 Sur couleur et peau, voir Grand-CLÉment 2011, 33-49.

17 Pour une étude plus approfondie des définitions philosophiques de la couleur, voir BradLEY 2009, chapitre 2.

18 Voir par exemple Platon, Charmide 167v-167d et Aristote, De l'âme, 418a27.

19 N.d.T. : l'auteur commente ici l'interprétation chromatique de l'expression homérique oinops pontos qu’implique la traduction consacrée "wine-dark sea ". Parmi les grandes traductions françaises, certaines comportent également un élément chromatique : " mer aux teintes lie-de-vin " (trad. P. Mazon, 1937) ou " mer aux couleurs de vin " (trad. P. Jaccottet, 1955). Ces dernières côtoient l'expression plus neutre "mer vineuse " de V. Bérard (1924), également privilégiée par F. Mugler (1989).

20 Pour une historiographie de cette question, voir Rutherford-Dyer 1983.

21 Voir déjà l'explication proposée par BÉNAKY 1915.

22 Voir Christol 2002. 
haut, Michael Clarke a suggéré que la catégorie oinops se référait en fait à des notions abstraites telles que " sombre comme le vin ", " délirant " ou " dionysiaque ", et que ses associations plus directes avec les propriétés du vin (non seulement son apparence visuelle, mais également son goût, son parfum, ses effets et ses dangers) contribuaient aux critères sémantiques délimitant son champ d'application de manière beaucoup plus significative que sa simple interprétation chromatique. Achille est ivre de chagrin et de rancune ; Ulysse a fait naufrage dans des eaux qui sont aussi profondes, intenses et périlleuses que le vin (et qui peuvent, à certains moments de la journée, présenter des longueurs d'ondes semblables) ; et ainsi de suite. La " couleur " oinops, pour autant que nous puissions la nommer ainsi, rappelait l'objet tout autant que son apparence ${ }^{23}$.

\section{Couleurs médicales}

Au cours des dernières années, d'importants travaux ont été réalisés - par des chercheurs français surtout - sur l'emploi de systèmes de classification faisant appel à la couleur dans les textes médicaux, du corpus hippocratique à Galien ${ }^{24}$. Afin de diagnostiquer les symptômes et de décrire la couleur de la peau, des yeux et des sécrétions de leurs patients, les experts médicaux avaient recours non seulement aux termes de couleur employés dans la poésie grecque, mais aussi, fréquemment, à des catégories issues d'objets de tous les jours, tels que le lait, les métaux, les pétales de fleurs, les lentilles ou le son. Une peau couleur de petit-lait (orōdēs), pour prendre un exemple, n'était pas seulement d'apparence blanc-jaunâtre, mais évoquait également une texture grumeleuse et peut-être aussi l'odeur caractéristique du pus ${ }^{25}$. On trouve également des exemples d'hommes et des femmes décrits comme ayant "la couleur de la sardine " ( $\tau$ ò $\chi \rho \tilde{\omega} \mu \alpha \dot{\alpha} \varphi v \tilde{\omega} \delta \varepsilon \varsigma)$ - une catégorie dont les qualités synesthésiques laissent peu de place à l'imagination $^{26}$. Les auteurs de textes médicaux de l'Antiquité établissaient en effet une relation étroite entre les couleurs du corps humain et les quatre humeurs - le sang, le phlegme, la bile jaune et la bile noire -, et celles-ci étaient justement, elles aussi, caractérisées par des propriétés tactiles - chaud-froid, humide-sec - et olfactives ${ }^{27}$.

\section{Couleurs physiognomoniques}

En troisième lieu, dans une branche des sciences apparentée à la médecine, la pensée physiognomonique de l'Antiquité a également eu recours à certaines de ces idées afin d'expliquer l'apparence, le caractère et le comportement des différents types ethniques ${ }^{28}$. Les effets de l'environnement physique sur l'apparence corporelle, et en conséquence sur le comportement, avaient déjà été explorés dans des textes médicaux tels que le traité hippocratique Airs, eaux, lieux, et on attribue parfois à Galien le mérite d'avoir intégré une théorie des humeurs aux principes physiognomoniques ${ }^{29}$. La catégorie Aethiops, par exemple, désignait littéralement

23 Sur les couleurs de la mer, voir Grand-Clément 2013.

24 Cf. Villard 2002, en particulier les contributions de L. Villard, V. Boudon et I. Boehm. Nous avons aussi abordé certains aspects de la relation entre couleur et médecine (BRADLEY 2009, I30-I35).

25 Hippocrate, Épidémies 7, 5, 9 et 7, 35, 2.

26 Voir Hippocrate, Des maladies de la femme 2, 110 et 2, 116, ainsi que VILlard 2002, 63.

27 À propos des subtilités de la perception sensorielle chez Galien, voir également SiEgEL 1970.

28 Pour une introduction à la physiognomonie dans l'Antiquité, voir BARTON 1994, chapitre 2 ; sur la relation entre médecine et physiognomonie, voir SASsi 1993.

29 Sur Galien et la théorie des humeurs appliquée à la physiognomonie, voir Evans 1941, 287-296. 
un "visage rendu foncé par le soleil " et était fréquemment employée par les Romains afin d'exprimer une sorte de distance physique et culturelle vis-à-vis des individus auxquels elle s'appliquait. En général, un tel visage avait été "coloré " (coloratus) ou "brûlé " (ustus) par la chaleur du soleil voisin : comme le disait Pline l'Ancien, ces peuples étaient nés avec une apparence brûlée et des cheveux crépus, tandis que ceux de la région du monde aux antipodes avaient une peau blanche et des cheveux blonds et raides. Sans surprise, Pline affirme que les Romains, situés entre les deux, avaient un teint "bien modéré » (color temperies) ${ }^{30}$. Vitruve fait état des mêmes polarités en employant la couleur comme un indicateur de qualités diverses, tant esthétiques que morales : les habitants du Nord, grands et bien hydratés, dont l'apparence (color) n'est pas privée d'humidité et qui ont par conséquent le teint pâle, les cheveux raides et roux, les yeux bleu clair et un sang abondant, s'opposent aux habitants du Sud, petits, brûlés et desséchés, avec leur teint basané, leurs cheveux crépus, leurs yeux noirs et leur sang pauvre $^{31}$. Sénèque est allé jusqu'à faire remarquer que les personnes blondes au teint rougeaud étaient particulièrement sujettes à la colère, parce que leur sang était pauvre et agité ${ }^{32}$. Dans ces contextes, la couleur ne participait pas seulement du spectre visuel : elle était un indicateur de la constitution physiologique intégrant (comme pour les théories médicales anciennes) diverses propriétés sensorielles.

La rougeur, une autre coloration physiognomonique, n'était habituellement pas associée à la race ou à l'origine mais constituait néanmoins un motif signifiant et récurrent de la littérature latine. La catégorie latine rubor, souvent traduite par "rougeur ", était une catégorie de l'expérience ancienne inextricablement liée au rougissement et à toutes ses implications physiologiques et morales ${ }^{33}$. On pouvait tout autant ressentir le rubor que l'on pouvait le voir : que l'on considère le fameux rougissement de Lavinia dans l'Énéide, le rubor des amants honteux dans la poésie élégiaque, ou celui de l'empereur Domitien dans les textes de Pline ou de Tacite, cette couleur est toujours chaude et fluide, tangible et incontrôlable ${ }^{34}$. Sénèque, dans l'une de ses Lettres à Lucilius, souligne le fait que les jeunes gens sont particulièrement sujets au rubor parce qu'ils ont davantage de chaleur corporelle et que leur enveloppe extérieure est molle ; leur sang, agité (incitatus) et instable (mobilis), leur monte facilement au visage ${ }^{35}$. À l'instar des autres exemples de couleurs somatiques évoqués plus haut, le rubor était imprégné de propriétés sensorielles dépassant le domaine visuel.

\section{La pourpre}

Le vêtement constituait l'un des moyens les plus parlants par lesquels le corps ancien pouvait faire usage de couleur et la donner à voir ${ }^{36}$. Les couleurs des habits provenaient nécessairement de teintures, et nous pourrions dès lors nous attendre à ce que cette expérience de la couleur ait opéré exclusivement dans le domaine visuel. Cependant, les teintures et les pig-

30 Pline, Histoire naturelle 2, 189. À propos de ces catégories (de manière générale), voir BALSDON 1979.

31 Vitruve, De l'architecture 6, 1, 3-4.

32 Sénèque, De la colère 2, 19, 5.

33 Nous avons déjà abordé la question du " rougissement " (" the colour blush") : cf. BradLey 2004.

34 À propos de Lavinia, cf. Virgile, Énéide 12, 64-71; pour les exemples tirés de l'élégie, cf. Ovide, Amours 2, 4, 33-50 ; à propos de Domitien, cf. Pline, Panégyrique 48, 4, et Tacite, Agricola 45, 2.

35 Cf. Sénèque, Lettres à Lucilius, 11, et Bradley 2009, 153-154.

36 Nous remercions Cambridge University Press de nous avoir accordé la permission de reproduire dans cette section certaines parties du chapitre 7 de notre ouvrage, Colour and Meaning in Ancient Rome (BradLEy 2009). 
ments utilisés avaient souvent eux-mêmes des propriétés olfactives et tactiles, de sorte que les vêtements pour lesquels ils avaient été employés prenaient part à une riche expérience polysensorielle. Les penseurs de l'Antiquité, parmi lesquels Théophraste, Vitruve et Pline l'Ancien, accordaient une grande importance à l'idée selon laquelle les teintures et les pigments, à l'instar des parfums, étaient, plutôt que de simples couleurs, des objets en soi, porteurs d'une gamme de propriétés esthétiques et de connotations : l'auteur du traité De coloribus (Sur les couleurs), par exemple, affirme le principe fondamental selon lequel toutes les choses teintes reçoivent leur couleur de l'objet qui les teint ${ }^{37}$.

Lorsque les Anciens portaient des robes de safran (crocotae) par exemple, ils le faisaient tout autant pour leur parfum que pour leur couleur distinctive, et les auteurs anciens ne manquèrent pas non plus de souligner leurs liens avec les fleurs et la féminité - que l'on pense aux notables romains efféminés des invectives de Cicéron, aux femmes parfumées de L'art d'aimer d'Ovide ou encore aux costumes safranés des prêtres eunuques auto-mutilés de Magna Mater $^{38}$. L'une des couleurs vestimentaires anciennes les plus odorantes était celle que donnait la teinture de pourpre marine (porphura en grec, purpura en latin), une couleur qui ornait les vêtements des monarques perses, ceux de la royauté hellénistique, ainsi que les attributs officiels de l'imperium romain. Cette teinture, qui existait en diverses nuances allant du rouge au noir en passant par le bleu, était célèbre pour son aspect remarquable ; mais elle possédait aussi une multitude d'autres propriétés qui frappaient non seulement les yeux, mais également les nez des observateurs anciens ${ }^{39}$. Face à la pourpre, certains penseurs de l'Antiquité étaient conscients des limites et des conséquences qu'impliquait le fait de privilégier une esthétique de la vision au détriment de tous les autres sens et d'ignorer les diverses qualités et propriétés de la riche culture matérielle de la Grèce et de Rome.

Pline l'Ancien, auteur d'une encyclopédie d'histoire naturelle du monde romain, fournit peut-être l'exemple le plus frappant d'une telle attitude. Son témoignage sur la pourpre débute sur les hauts-fonds du large de la côte phénicienne, au milieu du premier siècle de notre ère : c'est là que les pêcheurs locaux assuraient leur subsistance en pêchant les murex, mollusques qui nichaient par milliers dans les récifs affleurant au large de la Syrie et du Liban actuels. Dans le gosier de chacune de ces créatures, se trouvait une petite veine dont on tirait quelques gouttes du liquide qui, pendant des siècles, allait transformer les vêtements de l'élite babylonienne, égyptienne, perse, macédonienne et romaine. Malgré son histoire politique complexe et les discours profondément ancrés dans la culture consacrés à la teinture, Pline préféra revenir à ses fondamentaux. Son témoignage sur la pourpre (purpura en latin) prend place non pas dans un livre de son Histoire naturelle portant sur les pigments ou l'art du costume, mais dans celui ayant pour thème la mer : il s'agit d'un catalogue des phénomènes marins et des diverses

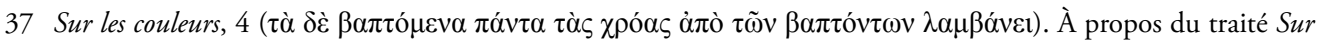
les couleurs, voir Gotтschalk 1964. Sur Théophraste, voir Baltussen 1998. Pour l'interprétation de la pourpre par Vitruve et pour Pline, voir Bradley 2009, 192-193 et chapitre 3 respectivement.

38 Cicéron, Sur la réponse des haruspices 21, 44 ; Ovide, L'art d'aimer 3, 179; Apulée, Métamorphoses 8, 27 ; Virgile, Enéide 11, 762-777 ; cf. 9, 614-617 (à propos des envahisseurs troyens efféminés vêtus de crocum et de purpura). Le safran est toujours une couleur vestimentaire caractéristique des disciples du mouvement Hare Krishna dans le monde entier. Sur les traditions et rituels Hare Krishna (d'un point de vue général), voir Dwyer, Cole 2007.

39 Sur l'histoire sur la pourpre en tant que symbole de prestige dans l'Antiquité, voir ReINHOLD 1976. Pour des approches diverses et variées de la teinture dans une perspective plus large, voir Longo 1998. Sur le rôle de la pourpre dans l'histoire de la couleur à Rome, voir BRADLEY 2009, chapitre 7. 
manières par lesquelles ceux-ci alimentaient le luxe romain. Le témoignage de Pline sur la pourpre constitue ainsi un rappel efficace de l'origine concrète des bandes de pourpre ornant les toges sénatoriales, des tenues triomphales et des autres costumes impériaux formant l'apanage du spectacle romain ${ }^{40}$.

Tel que décrit par Pline, le mollusque, qui avait en commun avec la teinture le nom de purpura, était une créature laide et répugnante, nourrie de vase, de limon et d'algues. Pline $(9,132)$ rapporte que les pêcheurs capturaient les murex en utilisant pour appâts des nasses remplies de coquillages dans lesquelles les mollusques étaient attirés par leur avidité (auiditas). Quelques paragraphes plus haut $(9,104)$, Pline déplore les périls dans lesquels les Romains étaient entraînés par leur appétit pour les produits de la mer - « la mer est des plus nocives pour l'estomac" (damnosissimum uentri mare est) - appétit qui, ajoutait-il, n’était rien en comparaison de l'avidité romaine pour les perles et la pourpre marine. En matière d'avidité - selon Pline du moins -, le mollusque fournissant la pourpre et le Romain vêtu de pourpre avaient beaucoup en commun. La purpura de Pline était dès lors un phénomène organique complexe doté d'une existence propre et associé à un ensemble d'idées morales et comportementales correspondant aux Romains qui la tenaient en si haute estime esthétique. Pline poursuit en décrivant le processus de fabrication de la teinture (133-134) sans épargner au lecteur des détails peu ragoûtants : la veine du mollusque était d'abord arrachée, puis mélangée à du sel et bouillie jusqu'à ce que la majeure partie de la chair se soit déposée. En faisant varier les mélanges et le temps d'ébullition, on obtenait différentes nuances. La plus estimée (summa laus) était celle qui rappelait le sang coagulé. Une méthode alternative consistait à diluer la teinture avec de l'urine humaine afin de produire " cette pâleur renommée " (ille laudatus pallor), c'est-à-dire que le teinturier pouvait tricher en s'épargnant le processus de saturation (saturitas fraudata) et ainsi économiser de l'argent. Pline conclut en ajoutant que les étoffes « dévorent " (esuriunt, un terme particulièrement fort) cette teinture diluée. Le mollusque, l'étoffe, le teinturier et le Romain élégant - tous ont en commun la même avidité (auaritia). Pline met tous les moyens en œuvre pour démontrer que la pourpre était investie de propriétés et de caractéristiques allant bien au-delà des considérations chromatiques.

Pline interrompt son témoignage sur le mollusque marin oriental nommé purpura pour formuler quelques remarques à propos de la purpura en tant que couleur romaine :

Fasces huic [purpurae] securesque Romanae uiam faciunt, idemque pro maiestate pueritiae est; distinguit ab equite curiam, dis aduocatur placandis, omnemque uestem inluminat, in triumphali miscetur auro. Quapropter excusata et purpurae sit insania ; sed unde conchyliis pretia, qui uirus graue in fuco, color austerus in glauco et irascenti similis mari?

"[Pour la purpura,] les faisceaux et les haches romaines ouvrent le chemin, et elle souligne aussi la dignité de l'enfance ; elle distingue le sénateur du chevalier et on la convoque pour s'assurer les faveurs des dieux. Elle donne de l'éclat à tout vêtement et, sur la robe du triomphateur, elle se mélange à l'or. Pour cette raison, on pourrait même excuser la folie de la pourpre - mais qu'est-ce donc qui justifie le prix des pourpres conchyliennes, avec la puanteur malsaine de leur teinture et leur couleur lugubre qui rappelle une mer sombre et courroucée? "

(Pline l'Ancien, Histoire naturelle 9, 127)

40 Cf. De la couleur, 4 ; un raisonnement semblable y est appliqué à la «pourpre marine " ( $\tau$ ò $\dot{\alpha} \lambda$ ov $\gamma \gamma \varepsilon \dot{\varsigma})$. 
Non sans provocation, Pline utilise le terme purpura tantôt dans le sens de la couleur des vêtements " pourpres ", tantôt dans le sens du mollusque : pour bien apprécier la première, il faut connaître la seconde. Plus haut, il introduisait son témoignage sur la purpura (105) en exprimant une frustration philosophique : "quel lien y a-t-il entre la mer et notre vêtement, entre les vagues, l'eau et notre tissu de laine " ? L'Histoire naturelle offre une démonstration magistrale de la manière dont on peut faire appel aux perceptions et aux catégories du sensible pour ordonner le monde et en tirer un savoir. Dès l'époque des présocratiques, les philosophes se sont querellés à propos de la relation complexe entre perception et savoir : dans quelle mesure pouvons-nous tirer de ce que nous voyons, entendons, sentons, goûtons et touchons autour de nous des informations sur le monde physique ${ }^{41}$ ? Certains - dont Platon - avaient défendu l'idée selon laquelle la perception ne permettait qu'une appréciation vague et peu fiable du monde ; d'autres considéraient que perception et connaissance étaient indissociables ${ }^{42}$. Pour Aristote et les Péripatéticiens, les couleurs étaient par essence des choses concrètes qui existaient dans le monde physique. Elles étaient à la base de la perception sensorielle et constituaient des balises renfermant une information cruciale sur le monde environnant. L'homme instruit avait pour tâche de mettre à profit toutes ses facultés afin de donner un sens à cet environnement. C'est une telle approche de la perception, profondément ancrée dans la philosophie stoïcienne, qui semble avoir prédominé à travers l'époque hellénistique et jusqu'à l'époque romaine.

Les préoccupations de Pline, dès lors, ne sont pas seulement celles d'un homme conservateur piqué dans ses valeurs morales. L'un de ses objectifs était de réaffirmer et de rétablir les principes physiques aristotéliciens : il voulait démontrer que l'épistémologie traditionnelle était minée par l'habitude romaine de prendre des bribes du monde - comme la purpura ou les pierres colorées -, de les tirer hors de leur contexte et de les apprécier pour leur seul aspect esthétique. De la même manière que l'on absorbe les principes de la nourriture pour se nourrir, poursuit-il, Pline entendait entraîner ses lecteurs à cultiver un savoir profond des objets qui dans leurs vies jouaient un rôle esthétique si important. Mais la catégorie purpura était dysfonctionnelle : le mollusque était immangeable, le vêtement, périssable (124), et la teinture, désagréable et malodorante (127). Selon son raisonnement, c'est en faisant appel au goût, au toucher et à l'odorat que l'on pouvait apprendre tout cela. Le jugement porté sur la pourpre par Pline, fondé sur ses principes fondamentaux, posait aux Romains une question rhétorique : pourquoi suscitait-elle un tel engouement? Sa mission consistait en deux points : premièrement, faire de purpura une catégorie appartenant au genre des mollusques; en second lieu, convaincre le lecteur que la " pourpre » constituait une expérience esthétique peu digne de l'indulgence romaine.

Pour des raisons similaires, d'autres auteurs du Haut-Empire romain s'efforcèrent également de rétablir le lien entre couleur et mollusque, et l'odeur de la teinture constituait un puissant rappel de son origine. Une génération après Pline, Martial, attaquant sur le mode satirique le penchant de ses contemporains pour le luxe exotique, décrit des lits imprégnés de la pourpre de Sidon à l'odeur forte $(2,16,3)$ et mentionne, parmi une liste de désagréments urbains courants, les vêtements empestant la teinture de murex $(1,39,32$ : olidae... uestes murice) ; ailleurs

41 Sur la philosophie de la perception dans l'Antiquité, voir Porter 2013. Diverses approches philosophiques de la perception des couleurs sont examinées dans BRADLEY 2009, chapitre 2.

42 À propos de la «synesthésie » en tant que mode de perception idéal selon Platon, voir Rosen 2013. 
$(4,4,6)$, il range la pourpre teinte deux fois parmi les pires odeurs nauséabondes de la ville, et dans une épigramme $(9,63)$, il affirme avec humour que les femmes de la haute société sujettes à la transpiration portaient des vêtements teints de pourpre non pas pour leur couleur, mais pour leur odeur couvrante (delectatur odore, non colore). Les traits spirituels de Martial et les commentaires culturels acérés de Pline réservaient ainsi le même traitement à cette teinture : tous deux étaient conscients que, dans la riche culture matérielle de la Rome du premier siècle, les objets et surfaces colorés étaient fréquemment isolés de leur contexte et arborés pour leurs seules qualités visuelles abstraites.

\section{Conclusion}

On pourrait recenser de nombreuses autres catégories de couleur auxquelles les Anciens avaient recours pour évoquer des qualités synesthésiques. La catégorie marmoreus (" de marbre »), par exemple, était couramment employée dans l'élégie latine pour décrire les nuques et les bras de jolies femmes, et évoquait aussi bien l'aspect froid et lisse des statues que la couleur blanche du marbre - par exemple, dans l'Appendix Vergiliana (Ciris, 256) : marmoreum tremebunda pedem... rettulit intra, "Toute tremblante, [Scylla] retira son pied "froid comme le marbre" sous [sa robe] $\aleph^{43}$. Dans un autre domaine du sensible, Martial décrit de manière imagée les conséquences d'un excès de bonne chère $(12,48)$, soit la goutte (carnifices pedes, " les pieds carnassiers") et un color sulphureus $(1,10)$. Il est clair que cette dernière expression, dans laquelle color a le sens de "carnation ", évoque autant la puanteur du soufre que sa couleur pâle ainsi que ses associations traditionnelles avec la mort ${ }^{44}$. Les cas d'usages polysensoriels de la couleur examinés dans cet article ne représentent que la pointe de l'iceberg : la littérature classique regorge d'exemples d'emplois de la couleur dans lesquels l'ecphrasis littéraire explore, dans un esprit ludique, les liens entre catégories visuelles et propriétés olfactives, tactiles, gustatives et même auditives du monde qu'elles décrivent. Une telle approche de la perception ancienne, que l'on pourrait qualifier de "synesthésique ", apporte un argument nouveau et conséquent à l'encontre des affirmations traditionnelles à propos de la vision prétendue lacunaire des couleurs chez les Grecs et les Romains ${ }^{45}$.

Les implications d'une telle expérience sensorielle complexe du monde sont nombreuses - pour le «spectacle » ancien, par exemple. L'expérience moderne du spectacle, comme la cérémonie du couronnement britannique ou l'ouverture des Jeux Olympiques, est habituellement dominée par la sphère du visuel (tout comme notre expérience de la musique est de plus en plus restreinte au domaine auditif) ; le spectacle ancien, en revanche, centré sur une esthétique complexe du monde matériel et sur l'identification précise et systématique de fonctions, d'objets, de groupes et d'individus, constituait souvent une expérience polysensorielle ; du moins était-elle ainsi décrite dans le discours de l'élite instruite ${ }^{46}$. Ovide, dans les Fastes, décrit de manière somptueuse la procession du premier janvier, emmenant les nouveaux magistrats,

43 À propos de marmoreus en tant que catégorie de couleur, voir BradLey 2006, 5-9.

44 À propos de la relation entre les sensations et la mort dans l'épopée latine, voir WaLTers 2013.

45 L'approche présentée dans cet article s'en tient avant tout aux aspects littéraires et philologiques, mais la couleur peut également être explorée, dans le domaine de l'art, en tant qu'indicateur de la matière : à ce sujet, voir entre autres Rouveret et al. 2006. Plus récemment, voir aussi CARASTRo 2009, qui regroupe des approches très variées de la couleur dans l'Antiquité.

46 Nous avons esquissé une comparaison entre l'ecphrasis entourant le couronnement de la reine Élizabeth II en 1953 et diverses descriptions du triomphe romain (Bradley 2009, 212-220). 
les sénateurs et le peuple vers le temple de Jupiter capitolin ; afin d'évoquer le renouvellement annuel de l'autorité romaine et la réaffirmation de la supériorité romaine impériale, il a recours à toute la gamme des sensations - vue, ouïe et odorat :

"Une aube heureuse se lève : sois favorable à nos vœux et à nos cœurs! Maintenant, de bons mots doivent être prononcés en un bon jour. Puissent nos oreilles être libres de poursuites judiciaires, et que les querelles insensées soient bannies sur-le-champ : vous, langues malveillantes, cessez de vous agiter! Voyez-vous comme l'air brille de feux parfumés et comme l'épi de Cilicie crépite sur les foyers ardents ? La flamme, de son propre éclat, frappe les ors des temples et répand une lumière dansante sur le toit du sanctuaire. Dans des vêtements purs, une procession monte vers la citadelle Tarpéienne, et le peuple a la même couleur que la fête. Maintenant les nouveaux faisceaux ouvrent le cortège, la pourpre nouvelle brille et la chaise d'ivoire qu'on voit de loin ressent de nouveaux poids. Les génisses insoumises au joug offrent leurs cous aux haches, génisses qu'a nourries l'herbe des pâturages des Falisques. Lorsque, depuis sa citadelle, Jupiter pose son regard sur le monde entier, rien ne s'offre à sa vue qui ne soit romain. Salut à toi, jour de joie, et reviens-nous toujours plus heureux, jour digne d'être célébré par un peuple maître du monde. »

(Ovide, Fastes I, 71-88)

Ce qu'Ovide présente ici est une immense expérience synesthésique, faisant appel à la vue, aux sons, aux odeurs et au toucher, de manière à offrir une appréciation entière et complète du spectacle ${ }^{47}$. En plus de l'aspect visuel - l'aube qui se lève, les flammes, les temples dorés, la pourpre magistrale, la chaise d'ivoire, etc. -, Ovide intègre toute la gamme des sens : les acclamations; les feux qui répandent une odeur (odoratis) en plus d'illuminer la scène ; l'épi qui crépite (sonat) sur les foyers ; l'éclat (nitor) des flammes de l'autel qui frappe (uerberat) le toit du temple ; les vêtements blancs (de la même couleur, souligne Ovide, que l'heureux dies candidus du premier janvier) arborant un aspect pur (intactis); la chaise d'ivoire qui ressent (sentit) le poids des nouveaux magistrats et les génisses indomptées offrant leurs nuques aux coups (ferienda $)^{48}$. Manifestement, il ne s'agit pas (comme nous aurions pu nous y attendre) d'une simple énumération de couleurs et d'expériences abstraites, mais d'un compte rendu riche et varié de visions, sons et odeurs spécifiques, affirmant le rôle du peuple romain en tant que maître du monde, maître des " choses " (res), comme le dit Ovide.

Cet article a examiné divers types de témoignages - des vers homériques aux écrits médicaux grecs, en passant par les fiancées rougissantes et les tissus malodorants. Ce serait aller trop loin que d'affirmer que la perception, dans l'Antiquité, était "synesthésique " au sens strict du terme ; nous pouvons toutefois identifier de nombreux exemples signifiants (et selon nos critères, légèrement inhabituels) d'expériences polysensorielles à l'œuvre dans la culture grécoromaine. La couleur est une notion que nous, Occidentaux modernes, rattachons habituellement au sens de la vue; comme nous avons tenté de le démontrer dans cet article, l'expérience ancienne de la couleur, parce qu'elle est directement liée aux objets de l'environnement plutôt qu'à une simple portion du spectre visible, pouvait quant à elle concerner l'odorat, le toucher, le goût et même le son. Pour les Grecs et les Romains, la couleur était à la fois une unité de

47 À propos des approches synesthésiques du cosmos par un autre poète latin, voir Volk 2013.

48 Pour des approches comparables, centrées sur l'expérience haptique, de la géographie chez Hérodote, voir Purves 2013. 
base de la perception, une source d'informations et de connaissances et un outil permettant d'appréhender finement le monde qui les entourait. Il s'agissait d'un critère de première importance pour décrire un objet, une personne, un bâtiment ou un paysage, de même qu'une catégorie permettant de jauger le caractère et la personnalité. Pour accomplir tout cela, souvent un seul sens ne suffisait pas.

Bien que l'on attribue souvent à Aristote le modèle de cinq sens distincts, celui-ci aurait probablement été surpris de se voir ainsi crédité. La théorie des sens développée par Aristote est en fait beaucoup plus complexe (et n'est pas toujours cohérente) : le philosophe est même allé jusqu'à suggérer - à cause des films d'atomes qui " touchent " l'œil - que tous les sens pouvaient être réduits au seul toucher ${ }^{49}$. Nous ne pouvons certes pas non plus prendre pour acquis, de quelque manière que ce soit, que la vision des sens par Aristote s'accordait avec celle des autres auteurs et penseurs anciens ; comme l'ont par exemple montré A. Clements et $\mathrm{M}$. Telò ${ }^{50}$, le genre de la comédie antique présente une approche bien différente des sens. Même à ce jour, parce qu'il est si difficile d'établir de manière définitive ce qu'est un " sens ", les neurologues ne s'entendent pas toujours sur leur nombre : affirmer qu'il existe cinq sens est, pourrait-on dire, un peu comme soutenir qu'il y a sept couleurs dans l'arc-en-ciel ${ }^{51}$. L'historien des sens, dès lors, se doit de demeurer ouvert d'esprit et d'accepter, lorsqu'il s'intéresse au monde ancien, l'éventualité d'un système de perception, de connaissance et de compréhension fort différent de celui qui nous est familier dans l'Occident moderne.

Mark Bradley

Department of Classics and Archaeology

University of Nottingham

University Park

Nottingham NG7 2RD

United Kingdom

Mark.Bradley@nottingham.ac.uk

49 La contribution d'Aristote au modèle des cinq sens a été étudiée par SoRABJi 1971 ; voir également JoHANSEN 1997. Voir aussi PorTer 2013.

50 Clements 2013 ; Telò 2013.

51 Lévolution culturelle d'un modèle fondé sur cinq sens a été exploré par VINGE 1975 ; les ambiguïtés entourant ce modèle sont analysées de façon convaincante par CLASSEN 1993, 2-11 (entre autres). 


\section{Bibliographie}

BALSDON 1979

J. Balsdon, Roman and Aliens, London 1979.

Baltussen 1998

H. Baltussen, "The Purpose of Theophrastus 'De sensibus' Reconsidered ", Apeiron 31.2 (1998), 167-199.

BARKER 1984

A. Barker, Greek Musical Writings Volume I: The Musician and his Art, Cambridge 1984.

Barton 1994

T. Barton, Power and Knowledge: Astrology, Physiognomics and Medecine under the Roman Empire, Ann Arbor (MI) 1994.

BEARd 2002

M. Beard, " Did Romans Have Elbows? Or: Arms and the Romans ", in P. Moreau (ed.), Corps Romains, Grenoble 2002, 47-59.

BÉNAKY 1915

N.-P. Bénaky, «Des termes qui désignent le violet dans l'Antiquité et de la signification des épithètes composées de iov 'violette' ", $R E G 28$ (1915), 16-38.

BradLEy 2006

M. Bradley, "Colour and Marble in Early Imperial Rome ", Proceedings of the Cambridge Philological Society 52 (2006), 1-22.

BRADLEY 2009

M. Bradley, Colour and Meaning in Ancient Rome, Cambridge 2009.

Campen 2008

C. von Campen, The Hidden Sense: Synaesthesia in Art and Science, Cambridge 2008.

Carastro 2009

M. Carastro (ed.), L'antiquité en couleurs : catégories, pratiques, représentations, Grenoble 2009.

\section{Christol 2002}

A. Christol, "Les couleurs de la mer", in L. Villard (ed.), Couleurs et vision dans l'antiquité classique, Mont Saint-Aignan 2002, 29-44.

Clarke 2004

M. Clarke, «The Semantics of Colour in Early
Greek Word-Hoard ", in L. Cleland and K. Stears (eds.), Colour in the Ancient Mediterranean World, Oxford, John and Erica Hedges, 2004, 131-139.

Classen 1993

C. Classen, Worlds of Sense: Exploring the Senses in History and Across Cultures, London 1993.

Clements 2013

A. Clements, " «Looking Mustard»: Greek Popular Epistemology and the Meaning of

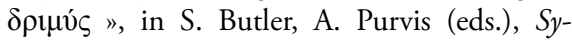
naesthesia and the Ancient Senses, Durham 2013, 71-88.

\section{Conklin 1955}

H. Conklin, "Hanonóo Color Categories ", Southwestern Journal of Anthropology 11.4 (1955), 339-344.

Coote 1992

J. Coote, " «Marvels of Everyday Vision»: The Anthropology of Aesthetics and the CattleKeeping Nilotes ", in J. Coote et A. Shelton (eds.), Anthropology, Art and Aesthetics, Oxford 1992, 245-273.

DWyer, Cole 2007

G. Dwyer, R. Cole (eds.), The Hare Krishna Movement: Forty Years of Chant and Change, London 2007.

Eco 1985

U. Eco, "How Culture Conditions the Colours We See ", in M. Blonsky (ed.), On Signs, Oxford 1985, 157-175.

\section{ECZET 2016}

J-B. Eczet 2016, « Perception et relation : l'expression du cattle complex par les Mursi ", in C. Fausto et C. Severi (eds), Paroles en images: écriture, corps et mémoires, Collection du programme Saint Hilaire, Open Edition Press, 35-58.

Evans 1941

E. Evans, "The Study of Physiognomy in the Second Century AD ", Transactions and Proceedings of the American Philological Association 72 (1941), 287-298. 
GeLl 1995

A. Gell, "On Coote's «Marvels of Everyday Vision»", Social Analysis 38 (1995), 18-30.

Gladstone 1858

W. E. Gladstone, Studies on Homer and the Heroic Age, vol. 3, Oxford 1858.

GotTschalk 1964

H. Gottschalk, "The De Coloribus and its Author ", Hermes 92.1 (1964), 59-85.

Grand-Clément 2011

A. Grand-Clément, La fabrique des couleurs: histoire du paysage sensible des grecs anciens, Paris 2011.

Grand-CléMent 2013

A. Grand-Clément, «La mer pourpre : façons grecques de voir en couleurs. Représentations littéraires du chromatisme marin à l'époque archaïque ", Pallas 92 (2013), 133-151.

Howes 2006

D. Howes, "Cross-talk between the Senses ", The Senses and Society 1 (2006), 381-390.

IRWIN 1974

E. Irwin, Colour Terms in Greek Poetry, Toronto 1974.

JOHANSEN 1997

T. K. Johansen, Aristotle on the Sense-Organs, Cambridge 1997.

LANDELS 1999

J. Landels, Music in Ancient Greece and Rome, London 1999.

LIENHARDT 1960

G. Lienhardt, Divinity and Experience: the Religion of the Dinka, Oxford 1960.

LoNGo 1998

O. Longo (ed.), La porpora: Realtà e immaginario di un colore simbolico (Convegno di studio di Venezia, 24-25 octobre 1996), Venezia 1998.

Maxwell-StUart 1981

P. G. Maxwell-Stuart, Studies in Greek Colour Terminology, vol. 1 : Glaukos; vol. 2 : Charopos, Leiden 1981.
PORTER 2013

J. I. Porter, "Why Are There Nine Muses ? ", in S. Butler, A. Purvis (eds.), Synaesthesia and the Ancient Senses, Durham 2013, 9-26.

Purves 2013

A. Purves, "Haptic Herodotus ", in S. Butler, A. Purvis (eds.), Synaesthesia and the Ancient Senses, Durham 2013, 27-41.

ReINHOLd 1976

M. Reinhold, The History of Purple as a Status Symbol in Antiquity, Bruxelles 1976.

REgan et al. 2001

B. Regan, C. Julliot, B. Simmen, F. Viénot, P. Charles-Dominique, J. D. Mollon, "Fruits, Foliage and the Evolution of Primate Colour Vision ", Philosophical Transactions: Biological Sciences 356/1407 (2001), 229-283.

Rosen 2013

R. M. Rosen, "Plato, Beauty and «Philosophical Synaesthesia" ", in S. Butler, A. Purvis (eds.), Synaesthesia and the Ancient Senses, Durham 2013, 89-102.

Rouveret, Dubel, NaAs 2006

A. Rouveret, S. Dubel, V. Naas (eds.), Couleurs et matières dans l'antiquité : textes, techniques et pratiques, Paris 2006.

RUTHERFORD-DYER 1983

R. Rutherford-Dyer, "Homer's Wine-Dark Sea ", Greece and Rome 30.2 (1983), 125-128.

SASSI 1993

M. Sassi, "Fisiognomica ", in G. Cambiano, L. Canfora, D. Lanza (eds.), Lo Spazio Letterario della Grecia Antica, Roma 1993, 431-478.

Schultz 1904

W. Schultz, Das Farbenempfindungssystem der Hellenen, Leipzig 1904.

SIEGEL 1970

R. Siegel, Galen on Sense Perception, New York 1970.

\section{Sorabji 1971}

R. Sorabji, «Aristotle on Demarcating the Five Senses ", Philosophical Review 80.1 (1971), 5579. 
TeLò 2013

M. Telò, "Aristophanes, Cratinus and the Smell of Comedy ", in S. Butler, A. Purvis (ed.), Synaesthesia and the Ancient Senses, Durham 2013, 53-70.

VILLARD 2002

L. Villard (ed.), Couleurs et vision dans l'antiquité classique, Mont-Saint-Aignan 2002.

VINGE 1975

L. Vinge, The Five Senses: Studies in a Literary Tradition, Lund 1975.
VolK 2013

K. Volk, "Manilius' Cosmos of the Senses ", in S. Butler, A. Purvis (eds.), Synaesthesia and the Ancient Senses, Durham 2013, 103-114.

WALTERS 2013

B. Walters, "Reading Death and the Senses in Lucan and Lucretius ", in S. Butler, A. Purvis (eds.), Synaesthesia and the Ancient Senses, Durham 2013, 115-126. 